\title{
Are general movements at 3-5 months correlated and compatible with the Bayley-III at 1,5-2 years age?
}

\author{
Aysu Kahraman ${ }^{1 \oplus}$, Ayşe Livanelioğlu², Özgün Kaya Kara², Murat Yurdakök ${ }^{3}$ \\ ${ }^{1}$ Department of Physiotherapy and Rehabilitation, Dr. Sami Ulus Maternity and Children's Health and Diseases Training and \\ Research Hospital; ${ }^{2}$ Department of Physiotherapy and Rehabilitation, Faculty of Health Sciences and ${ }^{3}$ Neonatology Unit, Department of \\ Pediatrics, Hacettepe University Faculty of Medicine, Ankara, Turkey.
}

\begin{abstract}
Since early intervention is important in risky infants, it is also important to identify developmental problem as early as possible. There are various assessment methods for this. One of them is the General movements assessment (GMs), and the other one is the Bayley Scales of Infants and Toddler Developmental, third edition (Bayley-III). The present study aimed to compare the neurodevelopmental outcomes and Bayley-III scores at the age of 1.5-2 years with fidgety GMs. One hundred and twenty-six infants ( 57 females, 69 males) were assessed by the GMs at the corrected 3-5 months and also by the Bayley-III at the age of 1.5-2 years. According to the GMs, 21 infants exhibited the absence of fidgety movements, six infants exhibited abnormal fidgety movements, and 99 infants exhibited normal fidgety movements. According to the Bayley-III, 19 infants' motor scores, 13 infants' language scores, and 18 infants' cognitive scores were low (-2SD). Various neurodevelopmental problems were identified in $25.4 \%$ of the infants. As a result, although in the present study the Bayley-III underestimates the rates of motor impairment, it was found to be moderately compatible with the GMs at 3-5 months ( $\mathrm{r}=0.4$, $\mathrm{p}<0.001)$. However, the GMs were better than the Bayley-III in predicting neurodevelopmental outcomes at the age of 1.5-2. Although the Bayley-III and GMs may be valuable tools for estimating the later outcomes of infants, care should be taken while interpreting their results.
\end{abstract}

Key words: fidgety movements, Bayley-3 infants and toddler developmental, neurodevelopmental outcome.

Because plasticity is rapid in the first two years, early diagnosis and treatment are essential in risky infants. Optimal treatment which is initiated at the early age for motor problems can reduce academic and psychosocial problems. ${ }^{1}$ There are various assessment methods for the early identification or prediction of infant problems. One of these is the assessment of general movements (GMs), the other one is the Bayley-3 Infants and Toddler Developmental Scale (Bayley-III). ${ }^{2,3}$

GMs are gross movements that include all parts of the body. ${ }^{2}$ They start during the 9th10th postmenstrual week. These movements

\footnotetext{
$凶$ Aysu Kahraman

aysum@hotmail.com
}

Received 6th September 2018; revised 20th March 2019; accepted 21st March 2019 decrease in the fourth and fifth months after birth and are replaced by goal-directed motor behaviors. ${ }^{4}$ The GM assessment is a good predictor for the identification of neurological impairments. ${ }^{5}$ As a result of the developmental changes of the nervous system, GMs differ in the three periods of preterm, writhing, and fidgety movements (FM). ${ }^{6} \mathrm{FM}$ are seen around three to five months' post-term. FM are small movements in all directions with moderate speed and variable acceleration in the neck, trunk, and limbs. ${ }^{7}$ While abnormal (AF), absent (F-), or sporadic FM indicate an increased risk of neurological dysfunction, normal FM $(\mathrm{F}+)$ have a high predictive value for normal development outcome. ${ }^{8}$

The Bayley-III is an assessment scale that is widely used to measure cognitive, language and motor conditions of infants. This scale 
assesses the development of infants and toddlers between 1 and 42 months of age. ${ }^{9}$ It is a discriminatory tool that compares a child's performance with other children. ${ }^{10}$

The present study aimed to compare the neurodevelopmental outcomes and Bayley-III scores at the age of 1.5-2 with fidgety GMs.

\section{Material and Methods}

This study was carried out between January 2012 - January 2016 with 57 females and 69 males, 126 infants in total, who applied to Department of Physiotherapy and Rehabilitation, Faculty of Health Sciences, Hacettepe University, Ankara, Turkey.

Infants with major malformations of the brain or other organs, infants with a chromosomal deformity, and infants whose parents no longer volunteered to participate or bring their infant for regular follow-up were discontinued from the study. This study was designed as a prospective trial of premature infants. The permission of the Hacettepe University Ethics Committee (GO 14/66-30) was received and written informed consent was obtained from the guardian of each participant.

The infants included in the study were evaluated according to Prechtl's general movement assessment. Infants aged between 9 - 17 weeks post-term were recorded for 3-5 minute while they were lying on supine position, awake and active. ${ }^{6}$ Evaluations of GMs were made by two experienced authors who had attended basic and advanced courses on GMs (basic: 2010, advanced: 2012). This was a blind study, the authors did not know anything about the infants' newborn period or previous problems during follow-up.

The Bayley-III was administered by another experienced author once for each participant at the corrected 18th or 24th months. The results were interpreted according to the composite score.

\section{Statistical analysis}

The data were analyzed using the IBM SPSS for Windows Version 16.0 software program. The Kolmogorov-Smirnov test was used to determine whether data had a normal distribution. Parametric tests were used for data with a normal distribution and non-parametric tests for data without a normal distribution. Mean, and standard deviation were calculated for numerical variables. Categorical variables were shown with numbers and percentages. The Kruskal-Wallis test was used to compare the data of more than two independent groups. On the other hand, the Mann-Whitney U test was used to compare the data of two independent groups. The Spearman correlation test was used to assess concordance between the data. Statistical results were interpreted at a 95\% confidence interval with a $\mathrm{p}<0.05$ (two-tailed) significance level.

\section{Results}

The clinical and demographic characteristics of infants are presented in Table I.

According to the GMs analysis, in the 3-5-month period, 99 infants exhibited $\mathrm{F}+$, six infants exhibited AF, and 21 infants exhibited F- movements. Of the infants, 94 with F+ had normal development, two had hypertonia, one had delayed motor development, and two had pervasive developmental disorder. One of the infants with AF had Cerebral Palsy $(\mathrm{CP})$, one had hypertonia, one had hearing loss, two had

Table I. Demographic and clinical characteristics of infants.

\begin{tabular}{lc}
\hline & Infant $(\mathrm{n}=126)$ \\
\hline Gestational age (Mean \pm SD) & $29.79 \pm 2.69$ \\
Birth weight (Mean \pm SD) & $1350.68 \pm 496.81$ \\
Jaundice (n) & $71(56.3 \%)$ \\
Respiratory distress syndrome (n) & $72(57.1 \%)$ \\
Pneumonia (n) & $26(20.6 \%)$ \\
Sepsis (n) & $44(34.9 \%)$ \\
Intraventricular hemorrhage (n) & $90(71.4 \%)$ \\
Bronchopulmonary dysplasia (n) & $45(35.7 \%)$ \\
\hline
\end{tabular}


Table II. Neurological outcome of infants.

\begin{tabular}{lcc}
\hline & Infant $(\mathrm{n}=126)$ & $\mathrm{GMs}(\mathrm{n}=126)$ \\
\hline Normal neurological outcome & $94(74.6 \%)$ & $94 \mathrm{~F}+$ \\
Cerebral Palsy & $16(12.7 \%)$ & $15 \mathrm{~F}-, 1 \mathrm{AF}$ \\
Hypotonia & $1(0.8 \%)$ & $1 \mathrm{~F}-$ \\
Hypertonia & $6(4.8 \%)$ & $2 \mathrm{~F}+, 1 \mathrm{AF}, 3 \mathrm{~F}-$ \\
Motor development delay & $2(1.6 \%)$ & $1 \mathrm{~F}+, 1 \mathrm{~F}-$ \\
Walking impairment & $1(0.8 \%)$ & $1 \mathrm{~F}-$ \\
Hear loss & $1(0.8 \%)$ & $1 \mathrm{AF}$ \\
Speech impairment & $1(0.8 \%)$ & $1 \mathrm{AF}$ \\
Pervasive developmental disorder & $4(3.1 \%)$ & $2 \mathrm{~F}+, 2 \mathrm{AF}$ \\
\hline
\end{tabular}

Table III. The comparison of Bayley-III Score and fidgety in infants.

\begin{tabular}{lccc}
\hline & $\begin{array}{c}\text { Bayley-III Motor } \\
(\text { Mean } \pm \text { SD })\end{array}$ & $\begin{array}{c}\text { Bayley-III Language } \\
(\text { Mean } \pm \text { SD })\end{array}$ & $\begin{array}{c}\text { Bayley-III Cognitive } \\
(\text { Mean } \pm \text { SD })\end{array}$ \\
\hline $\mathrm{F}+(\mathrm{n}=99)$ & $97.21 \pm 9.45$ & $98.15 \pm 8.85$ & $98.66 \pm 9.66$ \\
$\mathrm{~F}-(\mathrm{n}=21)$ & $72.67 \pm 23.15$ & $81.87 \pm 21.39$ & $76.48 \pm 21.65$ \\
$\mathrm{AF}(\mathrm{n}=6)$ & $86.17 \pm 23.20$ & $69 \pm 4.9$ & $76.17 \pm 19.03$ \\
\hline
\end{tabular}

pervasive developmental disorder, and one had a speech impairment. On the other hand, 15 of the infants with $\mathrm{F}$ - had $\mathrm{CP}$, one had hypotonia, three had hypertonia, one had walking impairment, and one had delayed motor development. The neurological outcomes of the infants are presented in Table II.

Since 63 of the infants could be followed up to 1.5 years old, and the remaining 63 up to 2 years old, the Bayley-III was applied at these ages. The Bayley-III motor scores were -2 SD below normal in 19 infants and four of these infants had normal development, 13 had $\mathrm{CP}$, one had hypertonia, and one had hypotonia. The BayleyIII motor scores were +1 SD above normal in 6 infants, and one of these infants did not hear, one had hypertonia, and four exhibited normal development. The Bayley-III language scores were -2 SD below normal in 13 infants, and five of these infants had $\mathrm{CP}$, one had a speech impairment, one had hypertonia, one did not hear, two had pervasive developmental delay, and three exhibited normal development. The Bayley-III language scores were +1 SD above normal in 7 infants, and one of them had pervasive developmental delay, one had hypertonia, one had $\mathrm{CP}$, and four exhibited normal development. The Bayley-III cognitive scores were-2SD below normal in 18 infants, and nine of them had $\mathrm{CP}$, one had hypertonia, one did not hear, two had pervasive developmental delay, one had motor retardation, one had a speech impairment, and three exhibited normal development. The Bayley-III cognitive scores were +1 SD above normal in 6 infants, and one of these infants had $\mathrm{CP}$, one had hypertonia, and four exhibited normal development.

While infants with F- had the lowest Bayley-III motor scores, infants with AF had the lowest Bayley-III language scores (Table III). Cognitive scores were found to be low in infants with both F- and AF. F+ infants' Bayley-III language and cognitive scores differed from those of $\mathrm{F}$ and AF infants. However, when it is examined from the Bayley-III motor scores point of view, there is a difference between $\mathrm{F}+$ and $\mathrm{F}-$, but not between $\mathrm{F}+$ and $\mathrm{AF}$.

Our results show that the GMs analysis and Bayley III scores are moderately compatible ( $\mathrm{r}=$ 0.4, $\mathrm{p}<0.001$ ). However, GMs were better than the Bayley III in predicting neurodevelopmental outcome at the age of $1.5-2$ years. 


\section{Discussion}

For risky infants is important to identify developmental problems as early as possible. The Bayley-III and GMs may be valuable tools for estimating the later outcomes of these infants.

The predictive value of AF is low. Infants with AF can show normal development or can have $\mathrm{CP}$ or minor neurological deficit. ${ }^{11,12}$ However, certain recent studies have shown that AF can be related to fine motor dysfunctions or autism spectrum disorder. ${ }^{13,14}$ Zappella et al. ${ }^{14}$ have reported that abnormal GMs were observed more frequently in infants who were diagnosed with autism spectrum disorder later. The fact that infants with AF had the lowest Bayley-III language score and a low cognitive score in our study shows that these infants may have more sensory influences than the motor. Our results indicate that $\mathrm{AF}$ movements, even if they do not predict $\mathrm{CP}$, are still consistent with a minor neurological impairment and behavioral, sensory, or cognitive developmental disabilities in the infants.

The presence of fidgety movements does not always show normal development. ${ }^{15}$ In the present study, all infants with $\mathrm{F}+$ did not have normal development at the age of 1.5-2 years. However, their Bayley-III scores were within normal limits. Furthermore, three infants with F- and mild CP had Bayley-III scores within normal limits. From this point of view, both tools do not have the ability to determine the developmental delay fully.

Fairbairn et al. ${ }^{16}$ showed that the Bayley-III performance at one year of age was not very successful at predicting the performance at three years of age. Anderson et al. ${ }^{17}$ reported that the Bayley-III had inflated scores and therefore was a poor predictor of cognitive and motor impairment. Spittle et al. ${ }^{3}$ indicated that although the Bayley-III motor scale at the age of two years could predict motor developmental retardation at four years of age in infants who were born earlier than 30 gestational weeks, it underestimated the rates of motor impairment.
Although similar results were obtained in our study, the Bayley is not a predictive tool, and it was developed as a discriminatory tool. Again, Peralta-Carcelen et al. ${ }^{18}$ found out that children's performance in the Bayley-III varies with age. In our study, the Bayley-III assessment was performed at the age of 1.5-2. All of these are the limitations of the present study.

As a result, although the Bayley-III underestimate the rates of motor impairment in our study, it was found to be moderately compatible with the GMs analysis at 3-5 months of age. However, GMs were better than the Bayley III in predicting neurodevelopmental outcomes at the age of 1.5-2 years. The Bayley-III and GMs may be valuable tools for estimating the later outcomes of infants, but care should be taken when interpreting their results. The later outcome could be decided more accurately if a more holistic assessment is performed with clinical findings, the duration of follow-up and the correct timing. Since the environment and the family can affect the development of a child during the time past, it should be kept in mind that none of the tests can succeed one hundred percent in the prediction of the later outcome.

\section{REFERENCES}

1. Jongmans MJ. Early identification of children with Developmental Coordination disorder. In: Sugden DA, Chambers ME, (eds). Children with Developmental Coordination Disorder. London: Whurr Publishers, 2005: 155-167.

2. Prechtl HFR. Qualitative changes of spontaneous movements in fetus and preterm infant are a marker of neurological dysfunction. Early Hum Dev 1990; 23: 151-158.

3. Spittle AJ, Spencer-Smith MM, Eeles AL, et al. Does the Bayley-III motor scale at 2 years predict motor outcome at 4 years in very preterm children? Dev Med Child Neurol 2013; 55: 448-452.

4. Lüchinger $A B$, Hadders-Algra $M$, van Kan $C M$, de Vries JI. Fetal onset of general movements. Pediatr Res 2008; 63: 191-195.

5. Bosanquet M, Copeland L, Ware R, Boyd R. A systematic review of tests to predict cerebral palsy in young children. Dev Med Child Neurol 2013; 55: 418-426. 
6. Einspieler C, Prechtl HF, Ferrari F, Cioni G, Bos AF. The qualitative assessment of general movements in preterm, term and young infants--review of the methodology. Early Hum Dev 1997; 50: 47-60.

7. Prechtl HF, Einspieler C, Cioni G, Bos AF, Ferrari F, Sontheimer D. An early marker for neurological deficits after perinatal brain lesions. Lancet 1997; 349: 1361-1363.

8. Einspieler C, Peharz R, Marschik PB. Fidgety movements - tiny in appearance, but huge in impact. J Pediatr (Rio J) 2016; 92(3 Suppl 1): S64-S70.

9. Bayley N. Bayley Scales of Infant and Toddler Development (3rd ed). San Antonio: Texas: Harcourt Assessment, 2006.

10. Heinemann KR, Hadders-Algra M. Evaluation of neuromotor function in infancy-A systematic review of available methods. J Dev Behav Pediatr 2008; 29: 315-323.

11. Brogna C, Romeo DM, Cervesi C, et al. Prognostic value of the qualitative assessments of general movements in late-preterm infants. Early Hum Dev 2013; 89: 1063-1066.

12. Einspieler C, Yang H, Bartl-Pokorny KD, et al. Are sporadic fidgety movements as clinically relevant as is their absence? Early Hum Dev 2015; 91: 247-252.

13. Einspieler C, Marschik PB, Milioti S, Nakajima Y, Bos AF, Prechtl HF. Are abnormal fidgety movements an early marker for complex minor neurological dysfunction at puberty? Early Hum Dev 2007; 83: 521-525.
14. Zappella M, Einspieler C, Bartl-Pokorny KD, et al What do home videos tell us about early motor and socio-communicative behaviours in children with autistic features during the second year of life-An exploratory study. Early Hum Dev 2015; 91: 569-575.

15. Hamer EG, Bos AF, Hadders-Algra M. Assessment of specific characteristics of abnormal general movements: does it enhance the prediction of cerebral palsy? Dev Med Child Neurol 2011; 53: 751756.

16. Fairbairn N, Galea C, Loughran-Fowlds A, Hodge A, Badawi N, Walker K. Prediction of three year outcomes using the Bayley-III for surgical, cardiac and healthy Australian infants at one year of age. Early Hum Dev 2018; 117: 57-61.

17. Anderson PJ, Burnett A. Assessing developmental delay in early childhood - concerns with the BayleyIII scales. Clin Neuropsychol 2017; 31: 371-381.

18. Peralta-Carcelen M, Moses M, Adams-Chapman I, Gantz M, Vohr BR; NICHD Neonatal Research Network; National Institutes of Health. Stability of neuromotor outcomes at 18 and 30 months of age after extremely low birth weight status. Pediatrics 2009; 123: e887-e895. 